Obesity, Food Restriction, and Implicit Attitudes to Healthy and Unhealthy Foods: Lessons Learned from the Implicit Relational Assessment Procedure

\author{
Ian McKenna ${ }^{1}$, Sean Hughes ${ }^{2}$, Dermot Barnes-Holmes ${ }^{2}$, Maarten De Schryver ${ }^{2}$, Ruth Yoder $^{3}$, \\ and Donal O'Shea ${ }^{3}$ \\ ${ }^{1}$ National University of Ireland Maynooth \\ ${ }^{2}$ Ghent University \\ ${ }^{3}$ St. Columcille's Hospital, Loughlinstown
}

Preparation of this paper was supported by a Government of Ireland Research Fellowship to SH and an Odysseus Type 1 grant (2015-2020) from the FWO to DBH. The empirical work was conducted as part of the first author's PhD conducted under the supervision of the third author and was funded by a Postgraduate Scholarship awarded to the first author by the Irish Research Council for Science, Engineering and Technology (now the Irish Research Council). IMK, Department of Psychology, National University of Ireland Maynooth, Ireland. RY and DOS, St. Columcille's Hospital, Loughlinstown, Ireland. SH, DBH, and MDS, Department of Experimental Clinical and Health Psychology, Ghent University, Belgium. Electronic mail should be sent to sean.hughes@ugent.be. 
IRAP Food Cognition 2

\begin{abstract}
It has been argued that obese individuals evaluate high caloric, palatable foods more positively than their normal weight peers, and that this positivity bias causes them to consume such foods, even when healthy alternatives are available. Yet when self-reported and automatic food preferences are assessed no such evaluative biases tend to emerge. We argue that situational (food deprivation) and methodological factors may explain why implicit measures often fail to discriminate between the food-evaluations of these two groups. Across three studies we manipulated the food deprivation state of clinically obese and normal-weight participants and then exposed them to an indirect procedure (IRAP) and self-report questionnaires. We found that automatic food-related cognition was moderated by a person's weight status and food deprivation state. Our findings suggest that the diagnostic and predictive value of implicit measures may be increased when (a) situational moderators are taken into consideration and (b) we pay greater attention to the different ways in which people automatically relate rather than simply categorize food stimuli.
\end{abstract}

Keywords: IRAP, Obese, Wanting, Hunger, Attitudes 
IRAP Food Cognition 3

Obesity, Food Restriction and Implicit Attitudes to Healthy and Unhealthy Foods: Lessons Learned from the Implicit Relational Assessment Procedure

The Western world is currently facing an obesity epidemic. Over the past three decades, obesity rates have exploded at an alarming rate (Finucane et al., 2011), to the point that one in three adults in the United States (Ogden, Carroll, Kit, \& Flegal, 2014) as well as one in five Europeans are currently overweight (WHO, 2013). As prevalence rates have grown so too have the health-related, socioeconomic, and psychological costs associated with this phenomenon (Finkelstein, Ruhm, \& Kosal, 2005; Wardle \& Cooke, 2005). With obesity comes an increased risk of type-II diabetes, adult heart disease, as well as several forms of cancer and premature morbidity (Reilly, et al., 2003; Wang, McPherson, Marsh, Gortmaker, \& Brown, 2011). In addition to its economic burden, obesity is also associated with a host of psychosocial problems, including anxiety, depression, and social discrimination (see Puhl \& Heuer, 2009; Sutin, Stephan, \& Terracciano, 2015; Wang et al., 2011). If we are to design effective interventions to ameliorate or even reverse this epidemic then research will need to elucidate those factors that contribute to the development and maintenance of this phenomenon.

One such factor appears to be food choice, and in particular, the excessive consumption of energy dense foods containing high levels of calories, sugars, and fat as well as the under consumption of nutrient dense foods such as fresh fruits and vegetables. Accumulating evidence suggests that obese individuals tend to consume higher levels of fats, sugars, and proteins than their normal-weight counterparts (e.g., Mai et al., 2011; McGloin et al., 2002; Nicklas, Yang, Baranowski, Zakeri, \& Berenson, 2003). Several authors have sought to explain this maladaptive pattern of behavior in terms of food-related evaluations. That is, obese individuals are thought to evaluate high caloric, palatable foods more positively than their normal weight peers, and this 
causes them to consume such foods, even when healthy alternatives are available (see Finlayson, Arlotti, Dalton, King, \& Blundell, 2011).

Yet when self-reported evaluations of healthy and unhealthy foods are examined no such evaluative biases tend to emerge. Obese individuals do not report increased positivity towards unhealthy foods, or even self-identify themselves more with those foods, than their normal weight counterparts (e.g., Creaynest, Crombez, Deforche, Tanghe, \& De Bourdeaudhuil, 2008; Craeynest, Crombez, De Houwer, Deforche, \& De Bourdeaudhui, 2006; Craeynest, Crombez, Haerens, \& De Bourdeaudhuil, 2007; see also Roefs, Herman, MacLeod, Smulders, \& Jansen, 2005; Veenstra \& de Jong, 2010). This absence of differential positivity towards healthy and unhealthy foods in obese and normal-weight populations may reflect a genuine similarity in evaluative tendencies between the two groups. However, two points are worth noting here. First, the above outcomes may have been influenced by social desirability concerns or impression management strategies. For instance, given the stigma associated with obesity (Puhl \& Heuer, 2009), and media portrayals linking it with excessive food consumption (Yoo \& Kim, 2012), obese individuals may be less inclined to publically endorse unhealthy foods in order to avoid further stigma and discrimination. At the same time, self-report measures can also fail to provide insight into food evaluations in situations where people lack the intention, awareness, or ability to control how they respond.

To overcome these limitations, a class of "indirect" procedures have emerged in recent times that do not depend on introspective access and which seek to minimize participants' control over their behavior. Whereas self-report measures of food evaluations can be classified as explicit measures that capture non-automatic instances of food-evaluation (e.g., food-evaluations that occur when participants have ample time and resources to reflect on, or have the intention to 
evaluate, a food item), implicit measures can be thought of as measures that register more spontaneous, automatic food-evaluations (e.g., food-evaluations that occur quickly or when participants do not have the intention to evaluate a food item; see Czyzewska, Graham, \& Ceballos, 2011; De Houwer, Teige-Mocigemba, Spruyt, \& Moors, 2009). Thus it may be that obese and normal weight individuals prefer healthy relative to unhealthy foods when their nonautomatic evaluative responses are assessed, but the former, unlike the latter group, display a stronger positivity bias towards unhealthy foods when their automatic evaluative responses are targeted ${ }^{1}$.

Once again, and contrary to expectations, a stronger positivity bias for unhealthy compared to healthy foods has not been observed. Several studies indicate that people automatically prefer healthy relative to unhealthy foods, regardless of whether they are obese or normal-weight (Craeynest et al., 2007; Roefs et al., 2005a; 2005b). Others report that obese individuals automatically evaluate healthy and unhealthy foods positively, suggesting a general enjoyment of eating rather a relative preference for one type of food over another (e.g., Craeynest et al., 2005; 2008; see also Craeynest et al., 2006). Still others have found that obese individuals tend to evaluate high-calorie sweet foods more negatively, and high-calorie nonsweet foods more positively, than their healthy counterparts (Czyzewska \& Graham, 2008; Czyzewska et al., 2011). Additional studies have found that although individuals with high BMI show no self-reported preference for high fat foods, they do show an automatic tendency to approach those very same foods (e.g., Veenstra \& de Jong, 2010). Therefore, the extent to which

\footnotetext{
${ }^{1}$ In-line with De Houwer and Moors (2010) we define a procedure as "direct" or "indirect" based on the way in which the measurement context is arranged to capture the behaviour of interest (e.g., verbal, speeded categorization of stimuli). We also define an outcome as either "explicit" or "implicit" based on the properties of the psychological attribute under investigation. Put simply, "implicit" and "explicit" refer to the operating conditions under which a psychological attribute influences outcomes rather than the procedure itself.
} 
obese and normal-weight individuals differ in their automatic and self-reported positivity towards unhealthy foods still remains something of a mystery.

\section{The Current Research}

We believe that two factors may help explain why implicit measures often fail to discriminate between the food-evaluations of obese and normal weight individuals. On the one hand, none of the above studies considered how situational moderators - such as restricted access to food - influenced automatic evaluations of healthy and unhealthy foods. This may be an important factor especially given that food-restricted participants tend to exhibit greater positivity towards food than their satiated counterparts (Hoefling \& Strack, 2008; Seibt, Häfner, \& Deutsch, 2007; Safford \& Scheffler, 2008). It may be that obese individuals do display stronger positivity towards unhealthy foods than their normal-weight counterparts, but only in situations where access to food has previously been restricted. In other words, when fully satiated, obese and normal weight participants may openly express (a) preferences for healthy relatively to unhealthy foods, or (b) a general preference for both types of food. Yet when obese individuals are subjected to certain situational stressors, such as restricted access to food, this pattern of food evaluations may shift, with unhealthy items quickly becoming their food of choice.

On the other hand, the type of indirect procedure employed may play an important role in discriminating the food evaluations of obese from normal-weight individuals. For instance, in nearly every study to date, researchers have relied on an Implicit Association Test (IAT; Greenwald, McGhee, \& Schwartz, 1998), Extrinsic Affective Simon Task (EAST; De Houwer, 2003) or an affective priming task (AP; Murphy \& Zajonc, 1993) to measure automatic foodrelated cognition. Although IAT, EAST and AP effects indicate the extent to which one set of 
concepts (e.g., 'unhealthy foods' and 'healthy foods') are related to a second set of concepts (e.g., good or bad adjectives), they do not provide any information about the specific manner in which those concepts are related. For instance, it may be that, for some individuals, the scores obtained from such procedures reflect beliefs that unhealthy foods are bad for them whereas for other individuals, those same scores might reflect beliefs that unhealthy foods prevent bad events such as negative thoughts and feelings from occurring (for related arguments see Remue, De Houwer, Barnes-Holmes, Vanderhasselt, \& De Raedt, 2013). Although both scores are derived from responding to stimuli such as 'unhealthy' and 'bad' they reflect fundamentally different ways in which these stimuli are related. Thus considering the different ways in which people (automatically) relate healthy and unhealthy food stimuli may provide even greater diagnostic and predictive information about their food-choices than simple food-valence categorization.

With this in mind, we set out to determine whether automatic and non-automatic responses to healthy and unhealthy foods vary according to one's weight status and food deprivation state. Across three separate studies we divided participants into two groups (normalweight and obese), manipulated their access to food and then administered a series of questionnaires and an indirect procedure known as the Implicit Relational Assessment Procedure (IRAP; Barnes-Holmes, Barnes-Holmes, Stewart, \& Boles, 2010; Vahey, Nicholson, \& BarnesHolmes, 2015). Unlike other tasks, the IRAP was designed to assess the speed and accuracy with which stimuli and events are related. During each trial, one stimulus is presented at the top of the screen (e.g., "I want to eat it now" or "I want to eat it later"), along with a second stimulus in the middle of the screen (e.g., images of healthy or unhealthy foods) and two response options at the bottom of the screen ("True" or "False"). By presenting specific combinations of stimuli together on each trial, and by requiring a particular response to be emitted quickly and accurately, the 
IRAP can assess how people relate those stimuli to one another in four different ways (e.g., $I$ want to eat unhealthy food now; I want to eat unhealthy food later; I want to eat healthy food now and I want to eat healthy food later $)^{2}$.

Based on previous work in this area, we did not expect obese and normal weight individuals to differ in their non-automatic food evaluations. That is, regardless of food restriction, we expected both groups to report (a) more positive evaluative responses for healthy over unhealthy foods or (b) a general positivity for both types of food. However, we did expect these two groups to differ in their automatic food-evaluations and for this to be moderated by prior food restriction. Specifically, we expected the automatic food evaluations of obese and normal-weight individuals to diverge from one another, with obese participants showing a relatively larger positivity bias towards unhealthy foods than their normal-weight counterparts whenever their access to food became increasingly restricted. Finally, and to the best of our knowledge, this is the first study to introduce the possibility that automatic evaluative responses toward healthy and unhealthy foods reflect individual specific relational responses. For cognitively orientated researchers these relational responses may be mediated by the formation and activation of food-related propositional beliefs rather than simple associations in memory.

\section{Experiment 1}

2 Note that unlike other measures of automatic responding the IRAP was derived from a basic theory of human language and cognition, known as Relational Frame Theory (RFT; Hayes, Barnes-Holmes, \& Roche, 2001). This theory constitutes a modern attempt to develop a behavioural and intensely pragmatic account that is best understood within the wider intellectual tradition of contextual behavioural science (see Zettle, Hayes, BarnesHolmes, \& Biglan, 2016). The core premise of RFT is that higher-level human cognition may be conceptualized as relational responses that occur within a given context. By relational responses we mean the action of responding to one stimulus in terms of another (e.g., "Unhealthy food is better than Healthy Food"). It has been argued that such responses may be mediated by propositions at the cognitive level of analysis (see Hughes, Barnes-Holmes, \& De Houwer, 2011). The important point here, however, is that the intellectual inspiration and theoretical context for the current research was RFT, and more specifically the idea that the automatic responses targeted by the IRAP involve brief and immediate relational responding to the various stimuli presented within the procedure itself. We shall return to this issue later in the General Discussion. 
Experiment 1 investigated the effects of food restriction on automatic evaluations of healthy and unhealthy foods among obese and normal-weight individuals. Half of the participants were asked to consume a large meal until full two hours prior to the study while the other half were provided with no such instructions. They were then administered a series of questionnaires along with an IRAP that was designed to target their automatic evaluations of healthy and unhealthy foods.

\section{Method}

\section{Participants}

Twenty three individuals ( 15 women) with severe obesity (age: $M=41.57$ years, $S D=$ 8.84; BMI: $M=50.04 \mathrm{~kg} / \mathrm{m}^{2}, S D=6.93$, range $=38-63$ ) were recruited on a voluntary basis from an Irish outpatient weight management clinic. Twenty four normal-weight individuals (11 women) (age: $M=20.92, S D=3.11$; BMI: $M=21.71 \mathrm{~kg} / \mathrm{m}^{2}, S D=1.89$; range: $18-24$ ) were also recruited on a voluntary basis from an undergraduate student population. Medical conditions were checked during a pre-screening interview, but none of the participants had to be excluded from the study on the basis of this interview.

\section{Materials}

Stimuli. Each participant was seated in front of a personal computer containing a version of the IRAP program. Six healthy (chicken salad, fruit, soup, green salad, nuts and a grilled fish dinner) and six unhealthy food images (hamburger and fries, donuts, chocolate, potato chips, ice-cream, and a steak dinner) were used during the IRAP and self-report measures. These images were drawn from an internet image database (www.fotosearch.ie) and selected based on descriptions of food stimuli used elsewhere in the literature (Roefs \& Jansen, 2002; Finlayson, King, \& Blundell, 2007). 
Self-Report Measures. We developed a screening questionnaire to assess the time since a participant's last meal, their present hunger state, and whether they had suffered from any gastrointestinal problems in the previous 48 hours. A food wanting questionnaire was also administered wherein healthy and unhealthy food stimuli were presented and participants were asked to rate how much they wanted to eat those foods using a 9-point scale that ranged from -4 (Later) to +4 (Now) with 0 as a neutral point. In a third task, participants completed the Eating Disorder Examination Questionnaire (EDE-Q5; Fairburn \& Beglin, 1994). This 28 item measure assessed for the presence and severity of specific eating psychopathology and was comprised of four subscales: restraint, weight concern, eating concern, and shape concern along with six pathological eating behavior items. Participants also completed the Balanced Inventory of Desirable Responding (BIDR; Paulhus, 1988). The BIDR is a 40 item scale designed to index a participant's propensity towards socially desirable responding. Half of the items assessed selfdeception while the remaining items assessed impression management. We included this scale to determine if socially desirable responding and impression management correlated with performances obtained from the self-reported questionnaires and IRAP. Finally, participants completed the 21-item Power of Food Scale (PFS; Lowe et al., 2009) to determine the psychological influence of the mere presence or availability of food. The PFS measured appetite for foods at three different levels: food availability, food presence, and food taste.

IRAP. The IRAP consisted of a minimum of one and a maximum of three pairs of practice blocks followed by a fixed set of three pairs of test blocks. Each block consisted of twenty four trials that presented one of two label stimuli ("I want to eat it now" or "I want to eat it later") at the top of the screen, a healthy or unhealthy food image in the middle of the screen, along with two response options ("True" or "False") at the bottom left and right-hand corners of 
the screen. This configuration of stimuli resulted in four different types of trials or "trial-types": Healthy Food-Now; Healthy Food-Later, Unhealthy Food-Now and Unhealthy Food-Later. The presentation of these trials, and allocation of the response options, were varied in a quasi-random order, such that each trial-type appeared an equal number of times within every block while the left-right positioning of the two response options varied across successive trials ${ }^{3}$.

At the beginning of the study participants were informed that a number of words and images would appear onscreen and that they would have to relate those stimuli in accordance with a general rule for responding. Specifically, during one block of trials, participants were required to respond as if they wanted to eat healthy foods now and unhealthy foods later. A response was deemed correct if it involved pressing "True" when presented with a healthy food item and "I want to eat it now" or an unhealthy food item and "I want to eat it later". A response was also deemed correct if participants pressed "False" in the presence of a healthy food and " $I$ want to eat it later" or an unhealthy food item and "I want to eat it now". During a second block of trials participants were required to respond in precisely the opposite way, acting as if they wanted to eat unhealthy foods now and healthy foods later. These two response contingencies were alternated across successive blocks of trials.

The IRAP commenced with a pair of practice blocks. Participants progressed from the practice to the test blocks when they met accuracy (at least $80 \%$ accuracy) and latency criteria (median latency of less than $3000 \mathrm{~ms}$ ) on a successive pair of practice blocks. Failure to meet these criteria resulted in re-exposure to another pair of practice blocks until participants either

3 We decided to relate food stimuli along a temporal dimension (now vs. later) in Experiment 1 given that food will always elicit some degree of wanting, even in situations where individuals are sated. For instance, after a large meal people may experience physical triggers indicating that they are sated but food wanting may still be evident - if only at a lower level than before (e.g., "I don't want any more cookies now but certainly want to eat them at some later point in time"). Thus we believed that statements such as "I want to eat now" and "I want to eat later" may correspond to a greater extent with everyday food behaviors than "I don't want to eat it at all". 
achieved those criteria or a maximum of three pairs of practice blocks were completed.

Participants who failed to meet the mastery criteria were provided with a short break and then reexposed to the IRAP once more (no participant failed to achieve the practice criteria during a second exposure). Once the above criteria were met, a fixed set of three test block pairs were administered.

\section{Procedure}

Upon arrival participants were welcomed by the researcher, seated in front of a computer, and provided with a brief description of the experimental agenda. Once they had provided their informed consent, they completed the food hunger questionnaire and an IRAP, followed by a food wanting questionnaire, the EDE-Q, BIDR and PFS. Note that half of the obese and normal weight participants were randomly assigned to an unrestricted food condition wherein they received no instructions to control their food intake prior to the study. The other half of participants were asked to restrict their food intake (i.e., they were instructed upon recruitment, and via a SMS message 24 hours prior to the study, to eat a large meal until full, finishing it two hours before the start of the experiment).

\section{Results}

\section{Analytic Strategy}

To determine whether (explicit and implicit) food wanting differed as a function of weight status and food restriction (both independent variables), we carried out linear mixed effects (LME) model analyses using the R package lme-4 (Bates, Maechler, Bolker, \& Walker, 2015). For each model, we defined as fixed effect variables the factors (effect coded) Food Type (healthy vs. unhealthy), Weight (Obese vs. Normal-Weight) and Food Restriction (Higher vs. Lower), their two-way interactions, and their three-way interaction. The grouping variable 
IRAP Food Cognition 13

'participant' was considered as a random factor. The reported $p$-values for the fixed effects are based on Type III ANOVA using a $\chi^{2}$-distribution.

In cases where three-way interactions were observed Generalized Wald tests $\left(\chi^{2}\right)$ on the variance/covariance matrices were used to test mean differences between the experimental conditions. We contrasted the following conditions for each of our outcome variables (IRAP and self-report scores): the estimated difference in mean Food Type, the estimated mean difference in Food Restriction as well as the two way interaction between Food Type and Food Restriction (i.e., the estimated differences between the mean differences of Food Type in both Food Restriction conditions) for normal-weight participants. A similar set of analyses were carried out for obese participants. We then contrasted these differences between normal-weight and obese participants (i.e., explored the three way interaction). Alpha was set at .05 for all statistical tests. Finally, we measured the extent to which the presence and severity of eating psychopathology (EDE-Q), socially desirable responding (BIDR), and the psychological impact of living in foodabundant environments (PFS) (all dependent variables) varied as a function of weight status and food restriction using a series of one-way ANOVAs and t-tests. The relationship between implicit (IRAP) and explicit (questionnaire) measures was also assessed using Pearson productmoment correlations.

\section{Data Preparation}

Time since last meal. Participants who were asked to restrict their food intake indicated that they consumed their last meal two hours prior to the study $(M=122.1, S D=12.2)$. Those provided with no such instructions indicated that they consumed their last meal considerably earlier that same day $(M=174.4, S D=102.8)$. Based on the data we labeled the former group as being 'lower in food restriction' and the latter group as being 'higher in food restriction'. 
Submitting time since last meal data to a 2 (Weight Status; Obese vs. Normal-Weight) x 2 (Food Restriction; Higher vs. Lower) one-way analysis of variance (ANOVA) revealed a main effect for Food Restriction, $F(1,46)=5.69, p=.02, \eta^{2}$ partial $=.18$, indicating that the two groups did indeed differ in the time since their last meal (no main or interaction effects emerged for Weight Status; all $p s>.5)$.

IRAP. The primary data obtained from the IRAP was response latency, defined as the time in milliseconds (ms) that elapsed from the onset of each trial to the first correct response emitted by the participant. To minimize contamination by individual differences associated with age, motor skills, and/or cognitive ability, response latency data were transformed into $D$-IRAP scores using an adaptation of Greenwald, Nosek and Banaji's (2003) D algorithm (see BarnesHolmes et al., 2011 for details of this transformation). For each participant we calculated a "healthy $D$-IRAP score" by collapsing the scores from the two healthy food trial-types into a single score. We then calculated an "unhealthy $D$-IRAP score" by collapsing scores from the two unhealthy food trial-types into a second score. In both cases, positive values indicate an automatic bias towards wanting to eat those foods now, negative scores indicate a bias towards wanting to eat those foods later while neutral scores indicate no preference for consuming those foods either now or later. Finally, we calculated a relative food preference score by subtracting healthy from unhealthy $D$-IRAP scores, with positive values indicating a preference for consuming unhealthy relative to healthy foods and negative values indicating the opposite.

Questionnaires. For each participant the twelve scores from the food wanting questionnaire were collapsed into two mean ratings, one for the healthy and another for the unhealthy food images. Positive values indicated that participants wanted to consume those items now while negative scores indicated that they wanted to consume those items later. Scores from 
the EDE-Q were collapsed into one (global) mean and four additional means, one for each of its four subscales (eating restraint, weight concern, eating concern, and shape concern). BIDR scores were averaged to create an overall score and also collapsed into two mean scores, one for the self-deception and impression management subscales. Finally, a PFS score was calculated by averaging the values obtained from that measure.

\section{Hypothesis Testing}

IRAP. Linear mixed effects analyses revealed a main effect for Food Type, $\chi^{2}(1)=3.95$, $p<.05$, as well as a marginally significant effect for Food Restriction, $\left(\chi^{2}(1)=3.58, p=.06\right.$. On the one hand, estimated mean IRAP scores were larger for the healthy $(M=0.32)$ compared to the unhealthy trial-types $(M=0.21)$. On the other hand, participants in the lower food restriction condition displayed a smaller automatic food bias $(M=0.20)$ than their counterparts in the higher food restriction condition $(\mathrm{M}=0.33)$. In short, neither weight status nor food restriction state had any influence on automatic food evaluations.

\section{Internal Consistency}

Split-half correlations for the $D$-IRAP scores obtained from odd and even trials were calculated using Spearman-Brown correlations. Internal consistency was $r=.45$ for the healthy and $r=.75$ for the unhealthy $D$-IRAP scores.

\section{Self-Report Measures}

Submitting scores from the food wanting questionnaire to a similar set of analyses revealed a main effect for Food Type, $\chi^{2}(1)=14.68, p<.001$, as well as a main effect for Food Restriction, $\chi^{2}(1)=5.75, p<0.05$. On the one hand, participants responded neutrally to healthy foods $(M=-0.03)$ and wanted to consume unhealthy foods at a later point in time $(M=-1.36)$. On the other hand, those in the higher food restriction group responded neutrally to foods $(M=$ - 
0.22) while their counterparts in the lower food restriction condition also wanted to consume food at a later point in time $(M=-1.19)$.

Consistent with the norm data reported by Mond, Hay, Rodgers, Owen, and Beumont (2004), the EDE-Q Global scores of the obese group $(M=3.17, S D=.95)$ significantly differed from those of the normal-weight group $(M=.91, S D=.60), F(46)=96.82, p<.001, \eta^{2}$ partial $=$ .68. A 2 (Weight) x 2 (Food Restriction) one way ANOVA indicated that obese individuals reported higher levels of pathological attitudes and behaviors towards food than their normalweight counterparts on the restraint $(M=2.41, S D=1.19$, vs. $M=1.17, S D=1.19), F(1,46)=$ $12.79, p=.001, \eta_{\text {partial }}^{2}=.23$, eating concern, $(M=2.36, S D=1.66$, vs. $M=.32, S D=.47), F(1$, $46)=32.22, p<.001, \eta^{2}$ partial $=.43$, shape concern, $(M=4.29, S D=1.14$, vs. $M=1.16, S D=$ $.78), F(1,46)=118.46, p<.001, \eta_{\text {partial }}^{2}=.73$, and weight concern sub-scales, $(M=3.64, S D=$ 1.25 , vs. $M=.98, S D=.74), F(1,46)=78.11, p<.001, \eta^{2}$ partial $=.65$ as well as in terms of their pathological food eating behaviors, $(M=23.87, S D=28.17$, vs. $M=1.96, S D=3.57), F(1,46)=$ 14.29, $p<.001, \eta_{\text {partial }}^{2}=.24$.

When overall and subscale BIDR scores were submitted to a similar set of analyses as above, the only significant effect to emerge was an interaction between Weight and Food Restriction on the Impression management subscale, $F(1,46)=4.03, p=.05, \eta_{\text {partial }}^{2}=.09$. Obese individuals' in the higher food restriction condition showed marginally higher levels of impression management $(M=7.42, S D=3.42)$ compared to their normal weight counterparts $(M$ $=4.42, S D=4.29), t(22)=1.89, p=.07$. However, in the lower food restriction condition, obese $(M=5.36, S D=2.77)$ and normal weight participants $(M=6.25, S D=2.42)$ both showed similar and lower scores than above, $t(21)=.82$. Obese and normal-weight individuals also produced similar Self-Deception, $(M=4.17, S D=3.2$, vs. $M=4.71, S D=3.0)$ and Global BIDR scores $(M$ 
$=10.7, S D=5.38$, vs. $M=10.04, S D=5.61)$. Finally, participants in the obese condition $(M=$ $66.09, S D=23.10)$ reported significantly higher appetite-related thoughts, feelings and motivations on the PFS compared to individuals in the normal-weight condition $(M=34.79, S D$ $=12.84), F(1,46)=32.76, p<.001, \eta_{\text {partial }}^{2}=.43$.

\section{Implicit-Explicit Correlations}

A correlation matrix was calculated in order to examine the relationship between the various self-report measures and $D$-IRAP scores. Analyses revealed non-significant correlations in all cases except between the unhealthy $D$-IRAP score and Global BIDR $(r=-.29, p=.05)$ as well as between the healthy $D$-IRAP score and self-reported wanting of healthy foods $(r=-.37, p=.01)$.

\section{Discussion}

Experiment 1 investigated the impact of food restriction on automatic evaluations of healthy and unhealthy foods among obese and normal-weight individuals. In-line with previous studies, we did not find sufficient evidence to conclude that automatic and non-automatic food evaluations differed as a function of a participant's weight or food restriction status. Participants demonstrated a general automatic food bias indicating that they wanted to immediately consume healthy and unhealthy foods, regardless of whether they were in the higher or lower food restriction condition. That said, self-report measures of food wanting did differ as a function of food type and prior restriction, with participants indicating that they wanted to consume unhealthy foods at a later point in time. Note that obese participants reported higher levels of pathological attitudes and behaviors towards food, propensity towards impression management and susceptibility to appetite-related thoughts, feelings, and motivations than their normal-weight peers. Therefore automatic and non-automatic food evaluations failed to discriminate between obese and normal-weight participants. 
IRAP Food Cognition 18

\section{Experiment 2}

Experiment 1 investigated whether participants automatically wanted to consume healthy and unhealthy foods, either now or at a later point in time. Contrary to our expectations, however, automatic food evaluations did not vary as a function of a person's weight status or restricted access to food. Given the current findings, and those elsewhere in the literature, it seems that obese and normal-weight participants may not differ in their implicit and explicit food wanting. We therefore decided to put food wanting to the side and explore the possibility that these two groups differ in other ways when it comes to healthy and unhealthy foods. Our thought was that the extent to which healthy and unhealthy foods automatically trigger thoughts and feelings about hunger may be a better predictor of a person's weight status than how much they want to consume those foods. For instance, during their day-to-day activities, obese individuals may have many more thoughts and feelings that center on their current hunger state relative to those that focus on their desire to consume a particular type of food. It may be that they also speak far more frequently about how hungry they are compared to how much they want to eat a given item, and do so at levels that exceed that of their normal-weight counterparts. If this hypothesis is correct, then presenting statements like "makes me hungry" together with pictures of healthy and unhealthy foods during the IRAP may serve to elicit responses that are far higher in probability than those seen when people respond to statements such as "I want to eat it". This would provide one explanation for why the wanting related statements in Experiment 1 failed to differentiate obese and normal-weight participants (i.e., because such statements elicit wanting related responses in the two groups with equal probability). Perhaps obese individuals focus far more on their self-discriminated hunger states than their normal-weight counterparts and that 
certain (unhealthy) foods tend to elicit these hunger-related thoughts and responses far more than others (healthy foods), especially when individuals are food restricted.

To test this possibility, we once again divided participants into two groups (higher versus lower food restriction) and exposed them to an IRAP and self-report procedures. The content of these procedures was modified to assess the extent to which healthy and unhealthy foods elicited feelings of hunger rather than wanting.

\section{Method}

\section{Participants}

Twenty five obese individuals (15 female) (age: $M=41.36$ years, $S D=9.91$; range: 25 56, BMI: $M=47.68 \mathrm{~kg} / \mathrm{m}^{2}, S D=8.31$, range $\left.=32-65\right)$ were recruited on a voluntary basis from an Irish outpatient weight management clinic. Thirty two normal-weight individuals (16 female) (age: $M=20.81$ years, $S D=1.36$, range, $18-23$; BMI: $M=22.55 \mathrm{~kg} / \mathrm{m}^{2}, S D=1.21$, range $=21$ 25) were also recruited on a voluntary basis from an undergraduate student population. Medical conditions were checked during a pre-screening interview and no participant was excluded based on this interview. Participants were randomly assigned to a food restriction conditions with gender counterbalanced across the two groups.

\section{Materials}

IRAP. The IRAP was similar to that employed in Experiment 1 with a single exception: the two label stimuli were changed from food wanting related statements ("I want to eat it now" and "I want to eat it later") to food hunger related statements ("Makes me feel hungry now" and "Does not make me feel hungry now").

Self-report measures. Participants were asked to complete a similar set of questionnaires as before (EDE-Q, BIDR, and PFS). This time, however, the food wanting scale was replaced 
with a food hunger scale which assessed how hungry healthy and unhealthy food images made participants feel. Each item on this scale was evaluated from -4 (Not Hungry) to +4 (Hungry) with 0 as a neutral point.

\section{Procedure}

The experimental sequence was similar to that outlined in Experiment 1. This time, however, participants completed an IRAP targeting the extent to which healthy and unhealthy foods automatically elicit hunger related responses. Thereafter they completed a set of self-report measures (hunger scale, EDE-Q, BIDR, and PFS) and were subsequently thanked, debriefed and dismissed.

\section{Results}

\section{Analytic Strategy}

The analytic strategy adopted here was similar to Experiment 1. Note, however, that a series of logistic regressions were also carried out to determine whether a person's weight status (dependent variable) could be predicted from their self-reported food hunger responses, presence and severity of eating psychopathology (EDE-Q), socially desirable responding (BIDR), or psychological impact of living in food-abundant environments (PFS), food restriction state, and automatic hunger responses to healthy and unhealthy foods ( $D$-IRAP scores) (all independent variables).

\section{Data Preparation}

Time since last meal. Participants who were asked to restrict their food intake indicated that they consumed their last meal two hours prior to the study $(M=122.8, S D=9.5)$. Those provided with no such instructions indicated that they consumed their last meal considerably earlier that same day $(M=231.7, S D=113.3)$. Based on the data we labeled the former group as 
being 'lower in food restriction' and the latter group as being 'higher in food restriction'. Submitting time since last meal data to a 2 (Weight Status; Obese vs. Normal-Weight) x 2 (Food Restriction; Higher vs. Lower) one-way analysis of variance (ANOVA) revealed a main effect for Food Restriction, $F(1,56)=23.84, p<.001, \eta_{\text {partial }}^{2}=.31$, indicating that the two groups did indeed differ in the time since their last meal (no main or interaction effects emerged for Weight Status; all $p s>.6)$.

IRAP. A healthy and unhealthy $D$-IRAP score was calculated in a similar manner as Experiment 1. Positive values indicate a bias towards responding to food stimuli as if they elicit hunger while negative values indicate the opposite (i.e., a bias towards responding to food stimuli as if they do not elicit hunger).

Questionnaires. The twelve scores obtained from the food hunger questionnaire were collapsed into two mean ratings, one for healthy and another for unhealthy food images. Positive values indicate that a food item elicited feelings of hunger while a negative score indicates the opposite. Preparation of data related to the EDE-Q, BIDR, and PFS was identical to that reported in Experiment 1. Finally, and prior to regression analyses, scores obtained from the IRAP and questionnaires were standardized.

\section{Hypothesis Testing}

IRAP. Linear mixed effects analyses revealed a main effect of Weight, $\chi^{2}(1)=14.92, p<$ .001 , a two-way interaction between Food Type and Weight, $\chi^{2}(1)=5.42, p=.02$, Food Type and Food Restriction, $\chi^{2}(1)=4.39, p=.04$, as well as the expected three-way interaction between Food Type, Food Restriction and Weight, $\chi^{2}(1)=4.56, p=.03$.

To explore this three-way interaction we carried out linear hypothesis tests which involved contrasting the means from Food Type and Food Restriction for obese and normal- 
weight participants. We then tested whether the differences observed within one group were different to those observed in the second group. Analyses revealed a main effect of Food Type for those in the normal-weight condition, $\chi^{2}(1)=6.71, p<.01$, a two-way interaction between Food Type and Food Restriction for those in the obese condition, $\chi^{2}(1)=7.92, p<.01$, and a two-way interaction between Weight and Food Type, $\chi^{2}(1)=5.42, p<.05$. Normal-weight participants displayed an automatic hunger bias for healthy foods regardless of whether they were in the lower $(M=0.31)$, or higher food restriction condition $(M=0.22)$. They also showed no evidence of an unhealthy food bias, regardless of whether they were in the lower $(M=0.11)$ or higher food restriction condition $(M=0.03)$. Their obese counterparts also displayed an automatic hunger bias for healthy foods. But this only emerged for those in the higher $(M=$ $0.49)$, and not in the lower food restriction condition $(M=0.21)$. At the same time, obese participants also displayed an unhealthy food bias regardless of whether they were in the lower $(M=0.52)$ or higher food restriction condition $(M=0.32)$.

Taken together, normal-weight individuals only showed an automatic hunger bias for healthy foods and no evidence for an unhealthy food bias regardless of their food restriction state. Obese individuals who had been deprived of food for roughly two hours (i.e., low food restriction condition) demonstrated an unhealthy (but no healthy) food bias while their counterparts who had been deprived of food for roughly 3-4 hours (i.e., higher food restriction condition) demonstrated an automatic (general) food bias.

\section{Internal Consistency}

The split-half correlations for the $D$-IRAP scores obtained from odd and even trials were calculated using Spearman-Brown correlations. Internal consistency was $r=.29$ for the healthy $D$-IRAP score and $r=.37$ for the unhealthy $D$-IRAP score. 
IRAP Food Cognition 23

\section{Self-Report Measures}

Submitting scores from the food hunger questionnaire to a similar set of linear effects

analyses yielded a main effect of Food Type, $\chi^{2}(1)=8.89, p<.01$, while linear hypothesis testing revealed that this main effect of Food Type was evident for those in the normal-weight, $\chi^{2}(1)=4.55, p<.05$, and obese conditions, $\chi^{2}(1)=4.39, p<.05$. Overall, participants did not self-report a hunger bias for unhealthy foods $(M=-0.13)$ but did report a slight hunger bias for healthy foods $(M=0.56)$. Thus, unlike the IRAP, the food hunger questionnaire failed to differentiate between participants' weight status even though the former and latter procedures both employed the same set of pictorial stimuli.

Obese individuals showed higher levels of pathological attitudes towards food than their normal-weight counterparts as measured by their EDE-Q Global and subscale scores as well as their pathological eating behavior scores (all $p s<.001)$. Submitting overall BIDR scores, as well as those from its two sub-scales, to a similar set of analyses only resulted in a significant interaction between Weight and Food Restriction on the Impression Management sub-scale, $F(1$, $56)=4.18, p<.05, \eta^{2}$ partial $=.07,($ all other $p s>.07)$. Obese individuals' in the higher food restriction condition $(M=7.86, S D=3.92)$ showed higher levels of Impression Management than their normal-weight counterparts $(M=4.56, S D=2.76), t(28)=2.69, p=.01$. This was not the case for those in the lower food restricted condition, with obese $(M=5.0, S D=3.46)$ and normal-weight individuals $(M=5.25, S D=2.84)$ responding in a broadly similar fashion. Finally, and overall, obese individuals $(M=66.09, S D=23.10)$ reported significantly higher appetite-related thoughts, feelings and motivations on the PFS compared to their normal-weight counterparts $(M=34.79, S D=12.84), F(1,56)=17.77, p<.001, \eta_{\text {partial }}^{2}=.25$.

\section{Implicit-Explicit Correlations}


A correlation matrix was calculated in order to examine the relationship between selfreport measures and $D$-IRAP scores. Analyses revealed significant correlations between the unhealthy $D$-IRAP score and eating restraint $(r=.32, p=.02)$, eating concern $(r=.39, p=.003)$, shape concern $(r=.38, p=.003)$, weight concern $(r=.41, p=.002)$, Global EDE-Q scores $(r=$ $.41, p=.001)$, and PFS scores $(r=.32, p=.02)$.

\section{Prediction of Weight Status from Implicit and Explicit Measures}

In five separate binary logistic regressions we set out to predict a person's weight status from their food restriction state as well as implicit and explicit measures. Step 1 of the model examined whether weight status could be predicted exclusively using explicit measures (i.e., from either self-reported hunger responses, PFS, Global EDE-Q, BIDR or PFS scores). Analyses revealed that PFS and Global EDE-Q scores were strong predictors of weight status, unlike selfreported hunger or BIDR scores, which failed to contribute to the model. Adding food restriction state at the second step did not contribute significantly to the model, with a similar pattern of results emerging as in Step 1. In Step 3, we included healthy and healthy $D$-IRAP scores and found that automatic hunger responses to unhealthy foods consistently predicted a person's weight status in ways that their automatic thoughts about healthy foods, or their non-automatic thoughts about (un)healthy foods did not (see Table 1).

\section{Discussion}

Results from Experiment 2 indicate that obese and normal-weight participants differ in their automatic hunger related responses to healthy and unhealthy foods and that this effect is moderated by their food restriction status. When normal-weight participants completed an IRAP assessing the extent to which certain foods elicit hunger they demonstrated a consistent bias for healthy foods and no evidence of an unhealthy food bias regardless of their food restriction state. 
A different picture emerged for their obese counterparts. Those who had been deprived of food for roughly two hours (i.e., low food restriction condition) demonstrated an unhealthy (but no healthy) food bias whereas their counterparts who had been deprived of food for roughly 3-4 hours (i.e., higher food restriction condition) demonstrated a general bias for both healthy and unhealthy foods. In other words, and unlike their normal-weight counterparts, obese participants displayed a consistent hunger bias towards unhealthy foods regardless of their food restriction state.

Although obese and normal-weight participants differed in their automatic food hunger (in a way that depended on their food restriction state) this was not the case for their selfreported food hunger suggesting a dissociation between the former and the latter outcomes. This is despite the fact that both the IRAP and self-report procedures employed the exact same set of pictorial stimuli. Interestingly, the unhealthy $D$-IRAP score correlated positively with pathological eating restraint on the EDE-Q scale, suggesting that increases in the automatic endorsement of unhealthy foods as eliciting hunger corresponds with increases in pathological dietary restraint. Finally, a series of logistic regressions revealed that participants pathological attitudes towards food (EDE-Q) and susceptibility to appetite-related thoughts, feelings and motivations (PFS scores) predicted their weight status. Even more interestingly, however, is the fact that automatic hunger related responses to unhealthy foods predicted a person's weight status in ways that automatic hunger responses to healthy foods, or self-reported hunger responses, did not.

\section{Experiment 3}

Experiment 2 demonstrated that obese and normal weight individuals differ in the extent to which unhealthy and healthy foods automatically trigger hunger related responses; that these automatic responses differ depending on the extent of prior food restriction; and that automatic 
hunger responses to unhealthy (but not healthy) foods predict a person's weight status. No such findings have ever been obtained when food wanting (Experiment 1) or the tendency to associate one's self (Craeynest et al., 2006) or other valenced stimuli (Roefs et al., 2005) with different types of food were assessed. Although obese and normal-weight individuals have been shown to differ in their automatic evaluations of savory and sweet unhealthy foods (Czyzewska \& Graham, 2008), this is the first instance where differences in how those same groups respond to healthy and unhealthy foods have been observed.

Given the novelty of our findings, and their theoretical and clinical implications, a replication seemed warranted. Therefore in Experiment 3 we divided a novel sample of obese and normal-weight participants into two groups (higher vs. lower food restriction) and exposed them to an IRAP and self-report procedures. Upon reflection we also realized that the IRAP statements used in Experiment 2 ("Makes me feel hungry now" and "Does not make me feel hungry now") required participants to respond to food-related stimuli in an 'all-or-nothing' fashion. Yet in many everyday situations - especially those where participants are not satiated food stimuli will likely elicit at least some degree of hunger, and thus the absolute nature of the above statements may have reduced the IRAP's ability to capture subtle differences between obese and normal-weight individuals. Therefore in our final study participants were asked to relate stimuli in a relative rather than absolute manner ("Makes me feel VERY hungry now" and "Makes me feel SLIGHTLY hungry now").

\section{Method}

\section{Participants}

Thirty two obese individuals ( 16 female) (age: $M=36.88$ years, $S D=9.76$; range: 18 56, BMI: $M=50.91 \mathrm{~kg} / \mathrm{m}^{2}, S D=13.86$, range $=32-98$ ) were recruited on a voluntary basis from 
an Irish outpatient weight management clinic. Forty two normal-weight individuals (20 women) (age: $M=20.76$ years, $S D=3.67$, range, $18-34$; BMI: $M=21.49 \mathrm{~kg} / \mathrm{m}^{2}, S D=2.39$, range $=18$ 27) were recruited on a voluntary basis from an undergraduate student population. Medical conditions were checked during a pre-screening interview and no participant had to be excluded on the basis of this interview.

\section{Materials}

IRAP. The IRAP was similar to that employed in Experiment 2 with a single exception: the two label stimuli were changed from absolute hunger statements ("Makes me feel hungry now" and "Does not make me feel hungry now") to relative hunger statements ("Makes me feel VERY hungry now" and "Makes me feel SLIGHTLY hungry now").

Self-report measures. A similar set of questionnaires were administered as before (food hunger, EDE-Q, BIDR). However, this time the food hunger questionnaire required participants to rate how hungry the healthy and unhealthy food images made them feel using a scale that ranged from -4 (Not Hungry) to +4 (Very Hungry) with 0 (Slightly Hungry) as a mid-point. The PFS scale was also replaced with a food liking questionnaire. Specifically, participants were asked to rate how much they liked or disliked the taste of healthy and unhealthy food items using a scale ranging from -4 (Not Palatable) to +4 (Palatable) with 0 (Neutral) as a mid-point.

\section{Procedure}

The experimental sequence was similar to that used in Experiment 2. Participants completed an IRAP targeting the extent to which healthy and unhealthy foods automatically elicited hunger related responses. This was subsequently followed by a set of self-report measures (food hunger scale, food liking scale, EDE-Q, and BIDR).

\section{Results}


IRAP Food Cognition 28

\section{Analytic Strategy}

The analytic strategy in Experiment 3 was similar to that of Experiment 2.

\section{Data Preparation}

Time since last meal. Participants who were asked to restrict their food intake indicated that they consumed their last meal two hours prior to the study $(M=124, S D=19.5)$. Those provided with no such instructions indicated that they consumed their last meal considerably earlier that same day $(M=256.2, S D=222.8)$. Based on the data we labeled the former group as being 'lower in food restriction' and the latter group as being 'higher in food restriction'. Submitting time since last meal data to a 2 (Weight Status; Obese vs. Normal-Weight) x 2 (Food Restriction; Higher vs. Lower) one-way analysis of variance (ANOVA) revealed a main effect

for Food Restriction, $F(1,69)=14.09, p<.001, \eta_{\text {partial }}^{2} .18$, indicating that the two groups did indeed differ in the time since their last meal (no main or interaction effects emerged for Weight Status; all $p s>.07)$.

IRAP. A healthy and unhealthy $D$-IRAP score was calculated as in Experiments 1-2. Positive values indicate a bias towards responding to food stimuli as if they elicit a high degree of hunger ("makes me feel very hungry") while negative values indicate the opposite (i.e., a bias towards responding to food stimuli as if they elicit a small degree of hunger; "makes me feel slightly hungry").

Questionnaires. Two mean evaluative ratings, one for healthy foods and another for unhealthy foods, were calculated from the food hunger questionnaire. These were scored so that positive values indicated that food made participants very hungry while negative values indicated that foods elicited no hunger. Two mean evaluative ratings were also obtained from the food liking questionnaire in a similar fashion, with positive values indicating that participants found 
the item palatable and negative values the opposite. EDE-Q and BIDR scores were obtained as before. All scores were standardized prior to regression analyses.

\section{Hypothesis Testing}

IRAP. Linear mixed effects analyses revealed a main effect for Weight, $\chi^{2}(1)=4.31, p=$ .04 , as well as the expected three-way interaction between Weight, Food Type and Food Restriction, $\chi^{2}(1)=19.86, p<.001$.

To explore this three-way interaction we carried out linear hypothesis tests in a similar manner to that described in Experiment 1. Analyses revealed a two-way interaction between Food Type and Restriction for those in the normal-weight, $\chi^{2}(1)=17.12, p<.001$, and obese groups, $\chi^{2}(1)=5.3, p<.05$. Normal-weight participants in the lower food restriction condition automatically responded to healthy foods as making them feel very hungry $(M=0.29)$, and showed no evidence of such an effect for unhealthy foods $(M=0.07)$. Their counterparts in the higher food restriction condition demonstrated a reversed pattern of responding such that they automatically responded to unhealthy foods as making them feel very hungry $(M=0.27)$ and showed no evidence of a healthy food bias $(M=0.01)$. Now consider the obese group. Those in the lower food restriction condition displayed an automatic bias towards unhealthy foods as making them feel very hungry $(M=0.38)$ and no such effect for healthy foods $(M=0.13)$. Yet their counterparts in the higher food restriction condition showed an automatic hunger bias for both healthy $(M=0.29)$ and unhealthy foods $(M=0.22)$.

In sum, normal-weight participants' automatically responded to healthy (but not unhealthy) foods as if they made them feel very hungry roughly two hours after their last meal. Yet this evaluative bias reversed roughly four hours after their last meal such that unhealthy (but not healthy) foods automatically elicited hunger related responses. Obese individuals produced a 
different pattern of outcomes. They automatically responded to unhealthy (but not healthy) foods as making them feel very hungry roughly two hours after their last meal. Similar to Experiment 2, those same participants demonstrated a general (automatic) hunger bias towards both healthy and unhealthy foods roughly four hours after their last meal.

\section{Internal Consistency}

The split-half correlations for the $D$-IRAP scores obtained from odd and even trials were calculated using Spearman-Brown correlations. Internal consistency was $(r=.60)$ for the healthy $D$-IRAP score and $(r=.35)$ for the unhealthy $D$-IRAP score.

\section{Self-Report Measures}

Submitting food hunger scores to linear mixed effects analyses revealed a main effect for Weight, $\chi^{2}(1)=13.28, p<.001$, and Food Restriction, $\chi^{2}(1)=13.22, p<.001$. On the one hand, obese participants indicated that foods did not make them hungry $(M=-0.98)$ while their normal weight counterparts indicated that foods made them slightly hungry $(M=0.37)$. On the other hand, participants in the lower restriction condition indicated that foods did not make them hungry $(M=-0.88)$ while their counterparts in the higher food restriction indicated that foods made them slightly hungry $(\mathrm{M}=0.42)$. Conducting similar analyses on the food liking data revealed no main or interaction effects for Weight or Food Restriction with participants evaluating healthy $(M=1.15)$ and unhealthy foods positively $(M=1.25)$. In-line with our previous findings, obese individuals reported higher levels of pathological attitudes towards food than their normal-weight counterparts as measured by EDE-Q Global scores, the four EDE-Q subscales and in terms of their pathological eating behaviors, (all $p s<.001$ ). Finally, a main effect for Weight was obtained on the overall BIDR scores, $F(1,73)=7.91, p=.006, \eta^{2}$ partial $=$ .10 , as well as Impression Management, $F(1,73)=5.46, p=.02, \eta^{2}$ partial $=.07$, and Social 
Desirability sub-scales of the BIDR, $F(1,73)=6.05, p=.02, \eta_{\text {partial }}^{2}=.08$. Obese participants demonstrated higher levels of impression management $(M=7.34, S D=4.37$, vs. $M=5.26, S D=$ $3.17)$ and socially desirable responding $(M=5.34, S D=3.59$, vs. $M=3.60, S D=2.59)$ than their normal weight counterparts.

\section{Implicit-Explicit Correlations}

A correlation matrix was calculated in order to examine the relationship between selfreport measures and $D$-IRAP scores. Significant correlations only emerged between the unhealthy $D$-IRAP score and EDE-Q shape concern $(r=.24, p=.04)$, and the EDE-Q weight concern scores $(r=.28, p=.02)$.

\section{Predictive Validity of Implicit and Explicit Measures}

Across six separate binary logistic regressions we sought to predict a person's weight status from their food restriction state, as well as implicit and explicit measures. Step 1 of the model examined whether weight status could be predicted exclusively using explicit measures (i.e., from either self-reported hunger or liking responses, Global EDE-Q or BIDR scores). Analyses revealed that explicit hunger for healthy and unhealthy foods, Global EDE-Q and BIDR scores all predicted weight status, unlike food liking scores, which failed to contribute to the model. Adding food restriction state at the second step did not contribute to model, with a similar pattern of results emerging as in Step 1. In Step 3, we included healthy and healthy $D$ IRAP scores. Once again, we found that automatic hunger responses to unhealthy foods consistently predicted a person's weight status in ways that their automatic thoughts about healthy foods, or their self-reported liking scores did not (see Table 2).

\section{Discussion}


Experiment 3 replicated the basic finding of Experiment 2. Obese and normal-weight participants differed in their automatic hunger related responses to healthy and unhealthy foods and these responses were once again moderated by their prior access to food. On the one hand, normal-weight participants' automatically responded to healthy (but not unhealthy) foods as if they made them feel very hungry roughly two hours after their last meal. Yet this evaluative bias reversed around four hours since their last meal, such that unhealthy (but not healthy) foods now elicited hunger related responses. On the other hand, obese participants automatically responded to unhealthy (but not healthy) foods as making them feel very hungry roughly two hours after their last meal. Yet, after roughly four hours, those same participants demonstrated a general (automatic) hunger bias towards both healthy and unhealthy foods. Regression analyses revealed that self-reported food hunger, pathological attitudes towards food (EDE-Q) and socially desirable responding (BIDR) predicted a person's weight status. Similar to Experiment 2, automatic hunger responses to unhealthy foods predicted a person's weight status in ways that their automatic hunger responses to healthy foods, or self-reported hunger responses did not.

\section{General Discussion}

In the current paper we argue that two factors may explain why implicit measures often fail to discriminate between the food-related cognitions of obese and normal weight individuals. The first concerns an important situational moderator which has largely been overlooked in the literature to date (i.e., food restriction). The second factor concerns the manner in which automatic food cognition is typically assessed. Previous studies have relied on the IAT, EAST and AP tasks which require participants to categorize foods with either the self (Craeynest et al., 2006) or valenced stimuli (Roefs et al., 2005). Yet even greater diagnostic and predictive information about food-choice may be obtained when we consider how people automatically 
relate rather than simply categorize food stimuli. With the above in mind, we manipulated clinically obese and normal-weight participants' access to food and then administered an IRAP along with several questionnaires. Across our studies we found that these two groups differed in their automatic responses to healthy and unhealthy foods; that this effect was moderated by their food restriction state; and that it emerged only when food hunger (rather than food wanting) responses were targeted.

For instance, when we targeted food wanting ("I want to eat it now" vs. "I want to eat it later") we found that obese and normal-weight participants both showed a general food bias towards healthy and unhealthy foods that was unaffected by their food restriction state. These findings mirror those seen elsewhere in the obesity literature and reinforce the idea that obese and normal-weight participants do not differ in terms of their automatic desire to consume healthy and unhealthy foods (e.g., Creaynest et al., 2006; 2007; 2008). Yet when we put food wanting to the side and focused our attention on the extent to which foods automatically elicit hunger related responses ("Makes me hungry now" vs. "Does not make me hungry now") an entirely different picture emerged. This time normal-weight participants displayed an automatic healthy food bias regardless of their food restriction state and no evidence of an unhealthy food bias at any point in time. This contrasted with the performance of their obese counterparts who indicated that unhealthy (but not healthy) foods automatically elicited hunger responses two hours after their last meal (lower food restriction). When obese participants were deprived of food for roughly 3-4 hours (i.e., higher food restriction condition) they now demonstrated a general bias for both healthy and unhealthy foods. Regression analyses also revealed that these automatic hunger responses for unhealthy foods predicted a person's weight status in ways that their automatic hunger responses for healthy foods, or self-reported hunger responses did not. 
Thus, to the best of our knowledge, this is the first study to demonstrate that clinically obese and normal-weight participants differ in their automatic cognitions regarding healthy and unhealthy foods.

Given the novelty of our findings we conceptually replicated our findings while requiring participants to emit a relative rather than absolute automatic hunger response to different types of food (“Makes me feel VERY hungry" vs. "Makes me feel SLIGHTLY hungry"). Our hope was that these minor modifications might capture subtle differences in the hunger related responses of obese and normal-weight individuals. Once again, we found that the two groups differed in their automatic hunger responses to healthy and unhealthy foods, and that these responses were influenced by a person's food restriction state. Specifically, normal-weight participants automatically responded to healthy (but not unhealthy) foods as if they made them feel very hungry roughly two hours after their last meal. Yet this evaluative bias reversed roughly four hours after their last meal, such that unhealthy (but not healthy) foods automatically elicited hunger related responses. In contrast, obese individuals automatically responded to unhealthy (but not healthy) foods as making them feel very hungry roughly two hours after their last meal. Similar to Experiment 2, obese participants demonstrated a general (automatic) hunger bias towards both healthy and unhealthy foods roughly four hours after their last meal. Once again, these automatic hunger responses significantly increased the predictive power of our model above and beyond the self-report measures alone.

\section{Open Questions and Future Directions}

Empirical implications. The current findings have empirical, theoretical, and clinical implications for the study of obesity. First, nearly all of the work on automatic food-related cognition has relied on indirect procedures that are either (a) incapable of distinguishing 
responses to healthy and unhealthy foods in a non-relativistic manner or (b) are unable to specify the different ways that participants automatically relate those stimuli (i.e., their automatic beliefs about food). For instance, previous studies using the IAT simply required people to categorize food with valenced (Food-Good) or self-related items (I-Food) while affective priming studies focused on the relationship between presentations of a prime (healthy food) and responses to an ensuing target (positive word) (Craeynest et al., 2007; Roefs et al., 2005). Our findings extend beyond this early work in several ways. They show that people are not limited to categorizing food stimuli automatically but that they can also emit beliefs about those stimuli under the conditions of automaticity. They show that the content of these automatic beliefs differ (e.g., "I want to eat healthy foods now" vs. "Unhealthy foods make me very hungry"), and that the endorsement of those beliefs depends on the context in which they are measured (food restriction) and the individuals involved (obese and normal-weight). Our findings also highlight a procedure (IRAP) that is capable of capturing these and other food-related beliefs in ways that elude many other indirect tasks (although see De Houwer, Heider, Spruyt, Roets, \& Hughes, 2015).

If we are correct, and people are capable of emitting automatic food-related beliefs in the blink of an eye, then several interesting possibilities emerge. Take, for instance, the content of the belief. Although we only considered food wanting vs. hunger beliefs there may be many others which better predict a person's weight status along with their food related behaviors. Future work could examine whether beliefs related to food-intake regulation ("I should eat this" vs. "I shouldn't eat this"), dietary self-monitoring ("I can stick to my diet" vs. "I cannot stick to my diet") or food addiction ("I really need to eat this" vs. "I can easily live without") can also be emitted automatically and guide people's eating, dieting, or addictive behaviors. Others could 
examine if the relational responses captured by the IRAP provide greater diagnostic and predictive information than scores obtained from other procedures, especially when it comes to the food behaviors of obese and normal-weight individuals. Future research will also need to determine whether, when, and to what extent automatic food-related responses predict actual food-choice behaviors. Increasing evidence suggests that dietary behaviors are often made quickly and in the absence of either intention or awareness. For instance, automatic evaluations of healthy (e.g., fruit) and unhealthy (e.g., candy) foods predict whether people will purchase or consume the former over the latter (Ellis, Kiviniemi, \& Cook-Cottone, 2014; Prestwich, Hurling \& Baker, 2011) as well as their likelihood of gaining weight across a one year period (Nederkoorn et al., 2010). Despite such findings, no published study has examined whether automatic food-related responses represent a useful determinant of food intake in clinical populations. It may be that automatic food-evaluations also guide the food purchasing, preparation and consumption decisions of obese and overweight individuals. Given our findings, it also seems likely that the relationship between automatic cognition and food decision making will be moderated by access to food (e.g., whether a person goes shopping on an empty/full stomach or opens the refrigerator when they are hungry vs. satiated). A logical next step would involve manipulating automatic food-related cognition and food choice in order to demonstrate that the selection of healthy and unhealthy foods in obese populations is also moderated by changes in how those foods are automatically evaluated. Similar analyses could also be carried out with restrained eaters as well as patients with bulimia or anorexia.

Theoretical implications. Second, our findings have theoretical implications. The majority of work on automatic food evaluations has started from the perspective that human cognition is carved into two conceptually distinct mental systems (i.e., associative linking vs. 
propositional reasoning) that function under different operating conditions (i.e., automatic vs. controlled) (see Gawronski, Brannon, \& Bodenhausen, in press; Hughes et al., 2011). Yet growing evidence suggests that humans can automatically relate stimuli in a wide number of ways and that the manner in which stimuli are related often leads to divergent outcomes on implicit and explicit measures. If this assumption is correct then people may automatically respond to healthy and unhealthy foods in more complex ways than initially thought and these responses may be mediated by the formation and activation of propositions rather than associations in memory (De Houwer, 2014; De Houwer et al., 2015). Therefore by targeting the specific ways in which people propositionally relate food stimuli under the conditions of automaticity we may be able to better predict their food purchasing, preparation, and consumption behaviors than before (e.g., "I want this but really shouldn't eat it" or "unhealthy foods stop me from feeling bad about myself').

The argument that implicit cognition is relational in nature is also consistent with conceptual developments that are taking place within Contextual Behavioral Science (Zettle et al., 2016). According to a theoretical account known as the Relational Elaboration and Coherence (REC) model (Barnes-Holmes, et al., 2010), researchers use terms such as 'implicit' or 'automatic cognition' to refer to relational responses that are (a) typically lower in their complexity than those not emitted under the conditions of automaticity and (b) that have been derived by the individual many times in the past (see Hughes, Barnes-Holmes, \& Vahey, 2012). Given a sufficient history of learning, and a measurement context capable of capturing those relations, implicit measures should reflect other types of relational responses. This would explain why we observed automatic effects for, and differences between, food wanting and hunger responses on the IRAP. The REC model also argues that relative differences in number of times 
a particular response has been derived by an individual may contribute to distinct outcomes on implicit measures. For instance, it may that the wanting IRAP in Experiment 1 and the hunger IRAPs in Experiments 2 and 3 captured relational responses that are broadly similar in their relational complexity but differ in the degree to which they have been derived by obese and normal-weight individuals in the past (for related findings see Nicholson \& Barnes-Holmes, 2012).

Clinical implications. Third, our findings, while certainly preliminary, may have clinical implications in the treatment and management of obesity. Take Experiments 2-3 where obese participants consistently displayed an automatic bias for unhealthy foods. Intervention targeting obesity typically tackle an individual's non-automatic food cravings, choices, and behaviors (Cooper, Fairburn \& Moors, 2004). But the current work suggests that efforts should also be taken to address automatic food biases as well. It's worth bearing in mind here that automaticity is not an all-or-nothing concept but rather one that is comprised of several sub-features such as speed, efficiency, awareness, intention, and control (see De Houwer \& Moors, 2012). Therefore future research could determine in what way the biases reported here were "automatic". For instance, if obese individuals are unaware of their automatic food biases then it may be useful for clinical interventions to orientate attention towards those biases. If this were the case then informing obese individuals that they are more likely to automatically experience unhealthy foods as hunger inducing several hours after eating their last meal could influence their subsequent dietary decisions. At the same time, it might be that people are fully aware of their automatic biases (Hahn et al, 2004) but are unable to control the impact of those biases on behavior, or that they are simply too afraid to report them out of fear of weight-based stigma or discrimination. If the latter is true then simply enhancing awareness of one's implicit food 
attitudes would not necessarily impact their effect on behavior. Rather clinical interventions would have to achieve greater openness and honesty with regard to food evaluations and increase the individual's sense of self-control over their food choice and consumption (for related arguments see Hofmann, Adriaanse, Vohs, \& Baumeister, 2013).

Limitations. When conducting the above work researchers could improve upon our studies in several ways. For instance, as one reviewer pointed out, the sample sizes utilized in our studies were relatively small and thus caution is warranted when interpreting our findings At the same time, the obese groups in Experiments 1-3 were comprised of individuals whose weight either fell into the moderately obese (BMI 30 to 35), severely obese (BMI 35 to 40) or very severely obese categories (BMI > 40). Although our data do provide a broad overview of the explicit and implicit food cognitions of moderately to very severely obese individuals, they do not allow us to systematically differentiate between people from these different weight categories. Future studies could therefore recruit a larger sample of obese participants and divide them into three clearly delineated groups (as a function of their weight class) in order to determine if our findings are consistent across weight categories or driven primarily by individuals from specific weight sub-classes (e.g., by those who are severely but not moderately obese). Such work could also compare the automatic and non-automatic food cognitions of these three classes with that of their overweight (BMI 25-30) and normal weight counterparts. Likewise, normal-weight and obese participants in Experiments 1-3 varied not only in terms of BMI but also age (and other relevant dimensions such as education level). As another reviewer pointed out, these differences may have an impact on participant's food habits and evaluations as well as sensitivity to food deprivation manipulations. Future work could investigate the aforementioned questions while carefully controlling for such factors. We found that a person's 
weight status was predicted by the extent to which unhealthy foods automatically elicited hunger. Future work could determine if these food-hunger responses are actually a better predictor of weight status (as well as food intake and approach-avoidance tendencies) than food wanting (Experiment 1) or the tendency to associate one's self, or other valenced stimuli, with different types of food. This work could also examine if predictive validity increases in-line with greater levels of food-restriction (e.g., do implicit healthy and unhealthy food biases of obese and normal-weight individuals also differ when they are fully satiated or when they are deprived of food for six, eight or more hours).

\section{Conclusion}

Overall, the current work adds to our understanding of automatic food-related cognition in clinical and non-clinical populations and suggests that the diagnostic and predictive value of implicit measures may be increased when the influence of situational moderators (food restriction) are taken into account, and our attention is shifted away from how people categorize food stimuli and towards the ways that people automatically relate stimuli. Whenever automatic food-hunger was assessed obese individuals consistently demonstrated an unhealthy food bias. This bias was often insensitive to their food restriction state as well as the subtle ways in which food stimuli were related. Those same individuals also demonstrated a healthy food bias but only when their access to food had been restricted for greater than two hours. This was not the case for their normal-weight counterparts who consistently showed a bias towards healthy foods irrespective of their food restriction state. This same group did demonstrate an unhealthy food bias in Experiment 3, but only when their access to food had been highly restricted and when they had to relate food stimuli in a more nuanced fashion ("Makes me VERY hungry" vs. "Makes me SLIGHTLY hungry"). Automatic hunger related responses (as measured by the IRAP) 
consistently predicted a person's weight status in ways that their self-reported hunger (Experiment 2) and liking (Experiment 3) did not, supporting the inclusion of the IRAP in future research on food attitudes in obese and normal-weight populations. 


\section{References}

Barnes-Holmes, D., Barnes-Holmes, Y., Power, P., Hayden, E., Milne, R., \& Stewart, I. (2006). Do you really know what you believe? Developing the Implicit Relational Assessment Procedure (IRAP) as a direct measure of implicit beliefs. The Irish Psychologist, 32(7), $169-177$.

Barnes-Holmes, D., Barnes-Holmes, Y., Stewart, I., \& Boles, S. (2010). A sketch of the Implicit Relational Assessment Procedure (IRAP) and the Relational Elaboration and Coherence (REC) model. The Psychological Record, 60(3), 10.

Cooper Z., Fairburn C.G., \& D.M Hawker (2004). Cognitive Behavioural Treatment of Obesity: A Clinicians Guide Behaviour Research and Therapy. New York, NY: The Guildford Press.

Craeynest, M., Crombez, G., Deforche, B., Tanghe, A., \& De Bourdeaudhuij, I. (2008). The role of implicit attitudes towards food and physical activity in the treatment of youth obesity. Eating behaviors, 9(1), 41-51.

Craeynest, M., Crombez, G., De Houwer, J., Deforche, B., \& De Bourdeaudhuij, I. (2006). Do children with obesity implicitly identify with sedentariness and fat food?. Journal of Behavior Therapy and Experimental Psychiatry, 37(4), 347-357.

Craeynest, M., Crombez, G., Haerens, L., \& De Bourdeaudhuij, I. (2007). Do overweight youngsters like food more than lean peers? Assessing their implicit attitudes with a personalized Implicit Association Task. Food Quality and Preference, 18(8), 1077-1084.

Czyzewska, M., \& Graham, R. (2008). Implicit and explicit attitudes to high-and low-calorie food in females with different BMI status. Eating behaviors, 9(3), 303-312. 
Czyzewska, M., Graham, R., \& Ceballos, N. A. (2011). Explicit and implicit attitudes to food. In Handbook of Behavior, Food and Nutrition (pp. 673-692). Springer New York.

De Houwer, J. (2003). The extrinsic affective Simon task. Experimental Psychology, 50(2), 77 -85 .

De Houwer, J. (2014). A propositional model of implicit evaluation. Social Psychology and Personality Compass, 8(7), 342-353.

De Houwer, J., Heider, N., Spruyt, A., Roets, A., \& Hughes, S. (2015). The Relational Responding Task: Toward a New Implicit Measure of Beliefs. Frontiers in Psychology (Cognition), doi: 10.3389/fpsyg.2015.00319

De Houwer, J., Teige-Mocigemba, S., Spruyt, A., \& Moors, A. (2009). Implicit measures: A normative analysis and review. Psychological bulletin, 135(3), 347.

Bates, D., Maechler, M., Bolker, B., \& Walker, S. (2015). Fitting Linear Mixed-Effects Models Using lme4. Journal of Statistical Software, 67(1), 1-48. doi:10.18637/jss.v067.i01.

Ellis, E. M., Kiviniemi, M. T., \& Cook-Cottone, C. (2014). Implicit affective associations predict snack choice for those with low, but not high levels of eating disorder symptomatology. Appetite, 77, 124-132.

Fairburn, C. G., \& Beglin, S. J. (1994). Assessment of eating disorders: Interview or self-report questionnaire?. International Journal of Eating Disorders, 16(4), 363-370.

Finkelstein, E., Ruhm, C., \& Kosal, K. (2005). Economic causes and consequences of obesity. Annual Review of Public Health, 26(3), 239-257.

Finlayson, G., Arlotti, A., Dalton, M., King, N., \& Blundell, J. E. (2011). Implicit wanting and explicit liking are markers for trait binge eating. A susceptible phenotype for overeating. Appetite, 57(3), 722-728. 
Finlayson, G., King, N., \& Blundell, J. E. (2007). Liking vs. wanting food: importance for human appetite control and weight regulation. Neuroscience \& Biobehavioral Reviews, 31(7), 987-1002.

Finucane, M. M., Stevens, G. A., Cowan, M. J., Danaei, G., Lin, J. K., Paciorek, C. J., ... \& Ezzati, M. (2011). National, regional, and global trends in body-mass index since 1980: systematic analysis of health examination surveys and epidemiological studies with 960 country-years and 9. 1 million participants. The Lancet, 377(9765), 557-567.

Gawronski, B., Brannon, S. M., \& Bodenhausen, G. V. (in press). The associative-propositional duality in the representation, formation, and expression of attitudes. In R. Deutsch, B . Gawronski, \& W. Hofmann (Eds.), Reflective and impulsive determinants of human behavior. New York, NY: Psychology Press.

Greenwald, A. G., McGhee, D. E., \& Schwartz, J. L. (1998). Measuring individual differences in implicit cognition: the implicit association test. Journal of Personality and Social Psychology, 74(6), 1464.

Greenwald, A. G., Nosek, B. A., \& Banaji, M. R. (2003). Understanding and using the implicit association test: I. An improved scoring algorithm. Journal of Personality and Social Psychology, 85(2), 197.

Hayes, S., Barnes-Holmes, D., \& Roche, B. (2001). Relational frame theory. A post Skinnerian account of human language and cognition. New York, NY: Kluwer Academic.

Hoefling, A., \& Strack, F. (2008). The tempting effect of forbidden foods. High calorie content evokes conflicting implicit and explicit evaluations in restrained eaters. Appetite, 51(3), 681-689. 
Hughes, S, \& Barnes-Holmes, D. (2013). A Functional Approach to the Study of Implicit Cognition: The IRAP and the REC model. In B. Roche \& S. Dymond. (Eds.). Advances in Relational Frame Theory \& Contextual Behavioural Science: Research \& Applications. New Harbinger Publications.

Hughes, S., Barnes-Holmes, D., \& De Houwer, J. (2011). The dominance of associative theorizing in implicit attitude research: Propositional and behavioral alternatives. The Psychological Record, 61(3), 6.

Hughes, S., Barnes-Holmes, D., \& Vahey, N. (2012). Holding on to our functional roots when exploring new intellectual islands: A voyage through implicit cognition research. Journal of Contextual Behavioral Science, 1(1), 17-38.

Juarascio, A. S., Forman, E. M., Timko, C. A., Herbert, J. D., Butryn, M., \& Lowe, M. (2011). Implicit internalization of the thin ideal as a predictor of increases in weight, body dissatisfaction, and disordered eating. Eating Behaviors, 12(3), 207-213.

Lowe, M. R., Butryn, M. L., Didie, E. R., Annunziato, R. A., Thomas, J. G., Crerand, C. E., ... \& Halford, J. (2009). The Power of Food Scale. A new measure of the psychological influence of the food environment. Appetite, 53(1), 114-118.

Mai, R., Hoffmann, S., Helmert, J. R., Velichkovsky, B. M., Zahn, S., Jaros, D., ... \& Rohm, H. (2011). Implicit food associations as obstacles to healthy nutrition: the need for further research. The British Journal of Diabetes \& Vascular Disease, 11(4), 182-186.

McGloin, A. F., Livingstone, M. B. E., Greene, L. C., Webb, S. E., Gibson, J. M. A., Jebb, S. A., Cole, T. J., Coward, W. A., Wright, A., \& Prentice, A. M. (2002). Energy and fat intake in obese children and lean children at varying risk of obesity. International Journal of Obesity, 26, 200-207. 
Mond, J. M., Hay, P. J., Rodgers, B., Owen, C., \& Beumont, P. J. V. (2004). Validity of the Eating Disorder Examination Questionnaire (EDE-Q) in screening for eating disorders in community samples. Behaviour Research and Therapy, 42(5), 551-567.

Murphy, S. T., \& Zajonc, R. B. (1993). Affect, cognition, and awareness: affective priming with optimal and suboptimal stimulus exposures. Journal of Personality and Social Psychology, 64(5), 723.

Nederkoorn, C., Houben, K., Hofmann, W., Roefs, A., \& Jansen, A. (2010). Control yourself or just eat what you like? Weight gain over a year is predicted by an interactive effect of response inhibition and implicit preference for snack foods. Health Psychology, 29(4), 389.

Nicholson, E., \& Barnes-Holmes, D. (2012). Developing an implicit measure of disgust propensity and disgust sensitivity: Examining the role of implicit disgust propensity and sensitivity in obsessive-compulsive tendencies. Journal of Behavior Therapy and Experimental Psychiatry, 43(3), 922-930.

Nicklas, T. A., Yang, S. -J., Baranowski, T., Zakeri, I., \& Berenson, G. (2003). Eating patterns and obesity in children. The Bogalusa heart study. American Journal of Preventive Medicine, 25, 9-16.

Ogden, C. L., Carroll, M. D., Kit, B. K., \& Flegal, K. M. (2014). Prevalence of childhood and adult obesity in the United States, 2011-2012. Journal of the American Medical Association, 311(8), 806-814.

Parling, T., Cernvall, M., Stewart, I., Barnes-Holmes, D., \& Ghaderi, A. (2012). Using the Implicit Relational Assessment Procedure to compare implicit pro-thin/anti-fat attitudes 
of patients with anorexia nervosa and non-clinical controls. Eating Disorders, 20(2), 127 $-143$.

Paulhus, D. L. (1988). Assessing self-deception and impression management in self-reports: The Balanced Inventory of Desirable Responding. Unpublished manual. University of British Columbia, Vancouver, Canada.

Prestwich, A., Hurling, R., \& Baker, S. (2011). Implicit shopping: Attitudinal determinants of the purchasing of healthy and unhealthy foods. Psychology \& Health, 26(7), 875-885.

Puhl, R. M., \& Heuer, C. A. (2009). The stigma of obesity: a review and update. Obesity, 17(5), 941-964.

Reilly, J. J., Methven, E., McDowell, Z. C., Hacking, B., Alexander, D., Stewart, L., \& Kelnar, C. J. (2003). Health consequences of obesity. Archives of Disease in Childhood, 88(9), $748-752$.

Remue, J., De Houwer, J., Barnes-Holmes, D., Vanderhasselt, M. A., \& De Raedt, R. (2013). Self-esteem revisited: Performance on the implicit relational assessment procedure as a measure of self-versus ideal self-related cognitions in dysphoria. Cognition \& Emotion, 27(8), 1441-1449.

Remue, J., Hughes, S., De Houwer, J., \& De Raedt, R. (2014). To be or want to be: Disentangling the role of actual versus ideal self in implicit self-esteem. PloS one, 9(9), e108837.

Roddy, S., Stewart, I., \& Barnes-Holmes, D. (2010). Anti-fat, pro-slim, or both? Using two reaction-time based measures to assess implicit attitudes to the slim and overweight. Journal of Health Psychology, 15(3), 416-425. 
Roefs, A., Herman, C. P., MacLeod, C. M., Smulders, F. T. Y., \& Jansen, A. (2005). At first sight: how do restrained eaters evaluate high-fat palatable foods?. Appetite, 44(1), 103 -114 .

Roefs, A., \& Jansen, A. (2002). Implicit and explicit attitudes toward high-fat foods in obesity. Journal of Abnormal Psychology, 111(3), 517.

Roefs, A., Stapert, D., Isabella, L. A. S., Wolters, G., Wojciechowski, F., \& Jansen, A. (2005). Early associations with food in anorexia nervosa patients and obese people assessed in the affective priming paradigm. Eating Behaviors, 6(2), 151-163.

Seibt, B., Häfner, M., \& Deutsch, R. (2007). Prepared to eat: How immediate affective and motivational responses to food cues are influenced by food deprivation. European Journal of Social Psychology, 37(2), 359-379.

Swinburn, B. A., Sacks, G., Hall, K. D., McPherson, K., Finegood, D. T., Moodie, M. L., \& Gortmaker, S. L. (2011). The global obesity pandemic: shaped by global drivers and local environments. The Lancet, 378(9793), 804-814.

Vahey, N. A., Nicholson, E., \& Barnes-Holmes, D. (2015). A meta-analysis of criterion effects for the Implicit Relational Assessment Procedure (IRAP) in the clinical domain. Journal of Behavior Therapy and Experimental Psychiatry, 48, 59-65.

Wang, Y. C., McPherson, K., Marsh, T., Gortmaker, S. L., \& Brown, M. (2011). Health and economic burden of the projected obesity trends in the USA and the UK. The Lancet, 378(9793), 815-825.

Wardle, J., \& Cooke, L. (2005). The impact of obesity on psychological well-being. Best Practice \& Research Clinical Endocrinology \& Metabolism, 19(3), 421-440. 
World Health Organization (2013). Country profiles on nutrition, physical activity and obesity in the 53 WHO European Region Member States. Methodology and summary (2013). Retrieved from http://www.euro.who.int/en/publications/abstracts/country-profiles-on -nutrition,-physical-activity-and-obesity-in-the-53-who-european-region-member-states. -methodology-and-summary-2013

Wright, S. M., \& Aronne, L. J. (2012). Causes of obesity. Abdominal imaging, 37(5), 730-732.

Yoo, J. H., \& Kim, J. (2012). Obesity in the new media: a content analysis of obesity videos on YouTube. Health Communication, 27(1), 86-97.

Zettle, R., Hayes, S., Barnes-Holmes, D., \& Biglan, T. (Eds.). (in press). Handbook of Contextual Behavioral Science. New York: Wiley. 


\section{Appendix A}

Table 1. Logistic regression analyses predicting weight status from self-report data (entered at step 1), food restriction state (entered at step 2), and $D$-IRAP scores (entered at step 3).

\begin{tabular}{|c|c|c|c|c|c|c|}
\hline \multirow{3}{*}{ Independent variables } & \multicolumn{2}{|c|}{ Model 1} & \multicolumn{2}{|c|}{ Model 2} & \multicolumn{2}{|c|}{ Model 3} \\
\hline & $\beta(\mathrm{SE})$ & Odds & $\beta(\mathrm{SE})$ & Odds & $\beta(\mathrm{SE})$ & Odds \\
\hline & & Ratio & & Ratio & & Ratio \\
\hline Explicit (Healthy) & $-.29(.28)$ & .75 & $-.32(.28)$ & .72 & $-.31(.31)$ & .73 \\
\hline Food Restriction & & & $.35(.55)$ & 1.42 & .61(.67) & 1.84 \\
\hline$D$-IRAP (Healthy) & & & & & $.46(.32)$ & 1.58 \\
\hline$D$-IRAP (Unhealthy) & & & & & $1.73(.55)^{* *}$ & 5.65 \\
\hline Model Chi-Square [df] & $1.16[1]$ & & 1.57[ & & 21.9 & \\
\hline$\%$ Correct Predictions & 61.4 & & 63.2 & & 78 & \\
\hline Nagelkerke $R^{2}$ & 0.03 & & 0.04 & & 0.4 & \\
\hline Explicit (Unhealthy) & $-.36(.28)$ & .69 & $-.37(.28)$ & .69 & $-.43(.34)$ & .65 \\
\hline Food Restriction & & & $.27(.55)$ & 1.32 & $.66(.68)$ & 1.91 \\
\hline$D$-IRAP (Healthy) & & & & & $.36(.33)$ & 1.44 \\
\hline$D$-IRAP (Unhealthy) & & & & & $1.77(.55)^{* * *}$ & 5.84 \\
\hline Model Chi-Square [df] & $1.77[1]$ & & $2.02[2]$ & & $22.60[4]$ & \\
\hline$\%$ Correct Predictions & 63.2 & & 59.6 & & 78.9 & \\
\hline Nagelkerke $\mathrm{R}^{2}$ & .04 & & .05 & & .44 & \\
\hline Global EDE-Q & $3.41(.89)^{* * *}$ & 30.19 & $3.54(.92) * * *$ & 34.44 & $5.34(1.94)^{* *}$ & 208.97 \\
\hline Food Restriction & & & $1.18(1.03)$ & 3.25 & $2.20(1.48)$ & 9.06 \\
\hline$D$-IRAP (Healthy) & & & & & $.73(.74)$ & 2.08 \\
\hline$D$-IRAP (Unhealthy) & & & & & $3.09(1.38)^{*}$ & 22.09 \\
\hline Model Chi-Square [df] & $48.47[1]$ & & $49.89[2]$ & & $61.41[4]$ & \\
\hline$\%$ Correct Predictions & 87.7 & & 93 & & 94.7 & \\
\hline Nagelkerke $\mathrm{R}^{2}$ & .77 & & .78 & & .88 & \\
\hline Global BIDR & $.37(.28)$ & 1.45 & $.36(.28)$ & 1.44 & $.71(.39)$ & 2.04 \\
\hline Food Restriction & & & $.17(.55)$ & 1.19 & $.26(.68)$ & 1.30 \\
\hline$D$-IRAP (Healthy) & & & & & $.41(.33)$ & 1.51 \\
\hline$D$-IRAP (Unhealthy) & & & & & $2.08(.63)^{* * *}$ & 7.96 \\
\hline Model Chi-Square [df] & $1.84[1]$ & & $1.94[2]$ & & $24.88[4]$ & \\
\hline$\%$ Correct Predictions & 61.4 & & 61.4 & & 82.5 & \\
\hline Nagelkerke $\mathrm{R}^{2}$ & .04 & & .05 & & .47 & \\
\hline PFS & $1.24(.38) * * *$ & 3.45 & $1.28(.39)^{* * *}$ & 3.60 & $1.15(.46)^{* *}$ & 3.16 \\
\hline Food Restriction & & & $.52(.63)$ & 1.68 & $.87(.75)$ & 2.39 \\
\hline$D$-IRAP (Healthy) & & & & & $.48(.34)$ & 1.62 \\
\hline$D$-IRAP (Unhealthy) & & & & & $1.39(.53)^{* *}$ & 4.05 \\
\hline Model Chi-Square [df] & $15.15[1]$ & & $15.83[2]$ & & $29.36[4]$ & \\
\hline$\%$ Correct Predictions & 77.2 & & 77.2 & & 86 & \\
\hline Nagelkerke $\mathrm{R}^{2}$ & .31 & & .33 & & .54 & \\
\hline
\end{tabular}

Note: * indicates that the coefficient is statistically significant at $\mathrm{p}<.05, * * \mathrm{p}<.01 * * * \mathrm{p}<.001$. Explicit (Healthy) $=$ Explicit hunger score for healthy foods. Explicit (Unhealthy) $=$ Explicit hunger score for unhealthy foods. Food Restriction $=$ Food restriction state PFS $=$ Power of food scale . 
Table 2. Logistic regression analyses predicting weight status from self-report data (entered at step 1), food restriction state (entered at step 2), and $D$-IRAP scores (entered at step 3).

\begin{tabular}{|c|c|c|c|c|c|c|}
\hline \multirow[b]{2}{*}{ Independent variables } & \multicolumn{2}{|l|}{ Model 1} & \multicolumn{2}{|c|}{ Model 2} & \multicolumn{2}{|c|}{ Model 3} \\
\hline & $\beta(\mathrm{SE})$ & Odds & $\beta(\mathrm{SE})$ & Odds & $\beta(\mathrm{SE})$ & Odds \\
\hline Explicit Hunger (Healthy) & $-.61(.26)^{*}$ & .55 & $-.71(.29)^{*}$ & .49 & $-.71(.29)^{*}$ & .49 \\
\hline Food Restriction & & & $-.49(.55)$ & .38 & $-.52(.59)$ & .59 \\
\hline$D$-IRAP (Healthy) & & & & & $.36(.28)$ & 1.44 \\
\hline$D$-IRAP (Unhealthy) & & & & & $.54(.28)^{*}$ & 1.72 \\
\hline Model Chi-Square [df] & $6.03[1]$ & & $6.83[2]$ & & 12.08[ & \\
\hline$\%$ Correct Predictions & 68.9 & & 73 & & 67.6 & \\
\hline Nagelkerke $\mathrm{R}^{2}$ & .11 & & .12 & & .20 & \\
\hline $\begin{array}{l}\text { Explicit Hunger } \\
\text { (Unhealthy) }\end{array}$ & $-.83(.27)^{* *}$ & .44 & $-.88(.29)^{* *}$ & .41 & $-.89(.29)^{* *}$ & .41 \\
\hline Food Restriction & & & $-.35(.53)$ & .71 & $-.39(.56)$ & .68 \\
\hline$D$-IRAP (Healthy) & & & & & $.33(.28)$ & 1.39 \\
\hline$D$-IRAP (Unhealthy) & & & & & $.62(.30)^{*}$ & 1.86 \\
\hline Model Chi-Square [df] & $10.75[1]$ & & $11.17[2]$ & & $16.44[4]$ & \\
\hline$\%$ Correct Predictions & 67.6 & & 68.9 & & 73 & \\
\hline Nagelkerke $\mathrm{R}^{2}$ & .18 & & .19 & & .27 & \\
\hline Explicit Liking (Healthy) & $-.17(.24)$ & .85 & $-.17(.24)$ & .85 & $-.17(.26)$ & .84 \\
\hline Food Restriction & & & $.01(.49)$ & 1.01 & $.01(.52)$ & 1.01 \\
\hline$D$-IRAP (Healthy) & & & & & $.29(.26)$ & 1.34 \\
\hline$D$-IRAP (Unhealthy) & & & & & $.57(.27)^{*}$ & 1.77 \\
\hline Model Chi-Square [df] & $.49[1]$ & & $.49[2]$ & & $6.18[4]$ & \\
\hline$\%$ Correct Predictions & 56.8 & & 56.8 & & 62.2 & \\
\hline Nagelkerke $\mathrm{R}^{2}$ & .01 & & .01 & & .11 & \\
\hline Explicit Liking (Unhealthy) & $-.17(.24)$ & .84 & $-.17(.24)$ & .48 & $-.16(.25)$ & .51 \\
\hline Food Restriction & & & $.05(.48)$ & 1.01 & $.07(.49)$ & 1.07 \\
\hline$D$-IRAP (Healthy) & & & & & $.28(.26)$ & 1.33 \\
\hline$D$-IRAP (Unhealthy) & & & & & $.57(.27)^{*}$ & 1.77 \\
\hline Model Chi-Square [df] & $.54[1]$ & & $.55[2]$ & & $6.17[4]$ & \\
\hline$\%$ Correct Predictions & 60.8 & & 56.8 & & 63.5 & \\
\hline Nagelkerke $\mathrm{R}^{2}$ & .01 & & .01 & & .11 & \\
\hline Global EDE-Q & $5.21(1.29)^{* * *}$ & 183.98 & $5.39(1.38)^{* * *}$ & 220.13 & $5.92(1.69) * * *$ & 392.11 \\
\hline Food Restriction & & & $.75(1.05)$ & 2.12 & $.56(1.15)$ & 1.75 \\
\hline$D$-IRAP (Healthy) & & & & & $.43(.61)$ & 1.54 \\
\hline$D$-IRAP (Unhealthy) & & & & & $1.05(.75)$ & 2.86 \\
\hline Model Chi-Square [df] & $73.81[1]$ & & $74.34[2]$ & & $76.98[4]$ & \\
\hline$\%$ Correct Predictions & 91.9 & & 91.9 & & 90.5 & \\
\hline Nagelkerke $\mathrm{R}^{2}$ & .85 & & .85 & & .87 & \\
\hline Global BIDR & $.71(.28)^{* *}$ & 2.03 & $.71(.28)^{* *}$ & 2.04 & $.81(.30)^{* *}$ & 2.26 \\
\hline Food Restriction & & & $.20(.49)$ & 1.23 & $.19(.53)$ & 1.21 \\
\hline$D$-IRAP (Healthy) & & & & & $.41(.28)$ & 1.51 \\
\hline$D$-IRAP (Unhealthy) & & & & & $.63(.29)^{*}$ & 1.88 \\
\hline Model Chi-Square [df] & 7.96 & & 7.89 & & 14.42 & \\
\hline$\%$ Correct Predictions & 66.2 & & 63.5 & & 68.9 & \\
\hline Nagelkerke $\mathrm{R}^{2}$ & .13 & & .14 & & .24 & \\
\hline
\end{tabular}

Note: * indicates that the coefficient is statistically significant at $\mathrm{p}<.05, * * \mathrm{p}<.01 * * * \mathrm{p}<.001$. Explicit (Healthy) $=$ Explicit hunger score for healthy foods. Explicit (Unhealthy) $=$ Explicit hunger score for unhealthy foods.

Restriction $=$ Food restriction state PFS $=$ Power of food scale . 